Meta

Journal des traducteurs

Translators' Journal

\title{
Quelques observations sur des traductions de Jabberwocky, de Lewis Carroll
}

\section{Denise Campillo et Mireille Lanctôt}

Volume 37, numéro 2, juin 1992

URI : https://id.erudit.org/iderudit/002192ar

DOI : https://doi.org/10.7202/002192ar

Aller au sommaire du numéro

Éditeur(s)

Les Presses de l'Université de Montréal

ISSN

0026-0452 (imprimé)

1492-1421 (numérique)

Découvrir la revue

Citer cet article

Campillo, D. \& Lanctôt, M. (1992). Quelques observations sur des traductions de Jabberwocky, de Lewis Carroll. Meta, 37(2), 214-231.

https://doi.org/10.7202/002192ar d'utilisation que vous pouvez consulter en ligne. 


\title{
QUELQUES OBSERVATIONS SUR DES TRADUCTIONS DE JABBERWOCKY, DE LEWIS CARROLL
}

Denise Campillo et Mireille LanCtôt

Université de Montréal, Canada

\author{
JABBERWOCKY \\ 'Twas brillig, and the slithy toves \\ Did gyre and gimble in the wabe: \\ All mimsy were the borogoves, \\ And the mome raths outgrabe. \\ "Beware the Jabberwock, my son! \\ The jaws that bite, the claws that catch! \\ Beware the Jubjub bird, and shun \\ The frumious Bandersnatch!" \\ He took his vorpal sword in hand: \\ Long time the manxome foe he sought- \\ So rested he by the Tumtum tree, \\ And stood awhile in thought. \\ And, as in uffish thought he stood, \\ The Jabberwock, with eyes of flame, \\ Came whiffling through the tulgey wood, \\ And burbled as it came! \\ One, two! One, two! And through and through \\ The vorpal blade went snicker-snack! \\ He left it dead, and with its head \\ He went galumphing back. \\ "And has thou slain the Jabberwock? \\ Come to my arms, my beamish boy! \\ o frabjous day! Callooh! Callay!" \\ He chortled in his joy. \\ 'Twas brillig, and the slithy toves \\ Did gyre and gimble in the wabe: \\ All mimsy were the borogoves, \\ And the mome raths outgrabe.
}

The Annotated Alice, pp. 191-197.

\section{LE JASEROQUE}

1 Il brilgue : les toves lubricilleux

2 Se gyrent en vrillant dans le guave, 
3 Enmîmés sont les gougebosqueux,

4 Et le mômerade horsgrave.

5 Garde-toi du Jaseroque, mon fils !

6 La gueule qui mord; la griffe qui prend!

Garde-toi de l'oiseau Jube, évite

8 Le frumieux Band-à-prend.

9 Son glaive vorpal en main, il va-

10 T-à la recherche du fauve manscant;

Puis arrivé à l'arbre Té-Té,

Il y reste, réfléchissant.

Pendant qu'il pense, tout uffusé

Le Jaseroque, à l'œil flambant,

Vient siblant par le bois tullegeais,

Et burbule en venant.

Un deux, un deux, par le milieu,

Le glaive vorpal fait pat-à-pan!

La bête défaite, avec sa tête

Il rentre gallomphant.

As-tu tué le Jaseroque?

Viens à mon cœur, fils rayonnais !

Ô jour frabbejeais! Calleau ! Callai !

Il cortule dans sa joie.

II brilgue : les toves lubricilleux

Se gyrent en vrillant dans le guave,

Enmîmés sont les gougebosqueux,

Et le mômerade horsgrave.

Traduction de Frank L. Warrin, The New Yorker, 10 janvier 1931, in The Annotated Alice, pp. 191-197.

\section{BREDOULOCHEUX}

Il était reveneure; les slictueux toves

Sur l'allouinde gyraient et vriblaient :

Tout flivoreux vaguaient les borogoves;

Les verchons fourgus bourniflaient.

«Au Bredoulochs prends bien garde, mon fils!

À sa griffe qui mord, à sa gueule qui happe!

Gare l'oiseau Jeubjeub, et laisse

En paix le frumieux, le fatal Pinçmacaque !»

Le jeune hornme, ayant ceint sa vorpaline épée,

Longtemps, longtemps cherchait le monstre manxiquais,

Puis, arrêté près de l'arbre Tépé,

Pour réfléchir un instant s'arrêtait.

Or, tandis qu'il lourmait de suffèches pensées,

Le Bredoulochs, l'œil flamboyant, Arrivait en barigoulant ! 
17 Une, deux ! une, deux ! Fulgurant, d'outre en outre,

18 Le glaive vorpalin perce et tranche : flac-vlan!

Il terrasse la bête et, brandissant sa tête,

Il s'en retourne, galomphant.

«Tu as tué le Bredoulochs!

Dans mes bras, mon fils rayonnois!

Ô jour frableux! callouh ! calloc !»

Le vieux glouffait de joie.

Il était reveneure; les slictueux toves

Sur l'allouinde gyraient et vriblaient;

Tout flivoreux vaguaient les borogoves; Les verchons fourgus bourniflaient.

Traduction de Henri Parisot, De l'autre côté du miroir et de ce qu'Alice y trouva, version bilingue Aubier-Flammarion, 1971.

\author{
$* * *$ \\ ... Jabberwocky is the greatest of all nonsense poems in English. \\ Martin Gardner \\ Je n'ai pas fait de traduction de Jabberwocky. J'ai essayé d'en traduire un fragment mais cela \\ m'a ennuyé. Je n'ai jamais aimé ce poème qui m'a toujours paru d'un infantilisme affecté...
}

Antonin Artaud, lettre à Henri Parisot.

Qu'il suscite admiration ou agacement, Jabberwocky est un poème très connu qui a été traduit plusieurs fois en français. Notre étude, de nature comparative, porte essentiellement sur deux traductions du poème. Elle ne veut pas prendre parti et s'inscrire dans un des deux grands courants qui animent la traductologie actuelle, attachement au texte source ou orientation vers le lecteur cible. Plus modestement, elle tentera de mettre en lumière, par l'examen minutieux de certains éléments du discours, les stratégies adoptées par les traducteurs.

Nous avons retenu comme outil d'analyse la connotation sémiotique, sans toutefois donner à cette notion valeur épistémologique; la simplicité de son mécanisme nous permet de structurer notre analyse d'une manière qui, tout arbitraire qu'elle soit, ouvre le texte à une manipulation ordonnée. Rappelons-en le principe ${ }^{1}$ : considérée comme un signe (signifiant/signifié ou connotant/connoté), elle utilise comme supports les divers systèmes qui structurent le texte (phonétique, lexical, syntaxique, stylistique, idéologique...). La mise en lumière des divers connotés permet une lecture plurielle du texte; le choix des connotants permet au traducteur d'utiliser à plein les possibilités de la compensation. L'analyse phonétique vient révéler la texture phonique du texte. Le travail de traduction est ainsi placé dans la perspective d'une intervention délibérée et critique.

Jabberwocky se situe à part dans la production poétique, puisqu'il entre dans le genre littéraire du nonsense, dont Lewis Carroll est un des plus illustres représentants dans la littérature enfantine: le plan sémantique, soumis à une volonté ludique, prime la recherche esthétique, qui lui est ici nettement subordonnée ; la facture est classique.

Les traductions étudiées sont les suivantes : celle de Frank L. Warrin, publiée en 1931 dans The New Yorker (notée FW), et la dernière version de Henri Parisot (HP), parue en 1971 (Parisot en avait publié deux autres en 1961 et 1963); nous évoquerons aussi d'autres traductions, plus fragmentaires (celles de Boris Vian et d'Antonin Artaud). 
Jabberwocky apparait dans le premier chapitre de Through the Looking-Glass. Alice, ayant traversé le miroir, feuillette un livre qui se trouve près d'elle et que tout d'abord elle ne peut lire. Puis, se souvenant qu'elle est passée de l'autre côté de la surface, elle tend le livre au miroir et peut enfin le déchiffrer. Il est tentant de l'imaginer le lisant à voix haute, puisque nous savons qu'elle parle et que les pièces du jeu d'échec ne l'entendent pas. Hélène Cixous, dans l'introduction à l'édition bilingue AubierFlammarion, nous y incite : «l'autre côté», dit-elle, désigne souvent la face sonore des choses ou des mots. Bonne raison de nous intéresser à l'aspect phonétique du poème.

Jabberwocky est marqué par le double ou l'image spéculaire : le poème se livre à Alice dans le miroir; le premier quatrain est double, c'est aussi le dernier du poème; reprise de ce quatrain au chapitre 6 du livre, avec explications de Humpty-Dumpty; redoublements de mots ou de syllabes; enfin, le héros semble double : fils / père.

La répétition s'élargit en lecture multiple : Carroll lui-même donne des explications diverses, au fil du temps, pour les mots qu'il a inventés.

Le poème se compose de sept quatrains, le septième étant une répétition du premier (nous parlerons du quatrain 1-7). Dans chaque quatrain, on trouve trois tétramètres iambiques (à l'exception des vers 7,11 et 22) suivis d'un trimètre (iambique dans les quatrains 2 à 6 ).

Cette symétrie formelle est reprise en écho par le fond. Nous pouvons résumer Jabberwocky comme suit : un refrain qui ouvre et ferme le poème (qu. 1-7), lui donne un ton mystérieux, introduit un élément temporel, puis une narration :

qu. 2 - mise en garde du père;

qu. 3 - départ et quête;

qu. 4 - apparition du monstre;

qu. 5 - bataille et victoire;

qu. 6 - retour et récompense.

Jabberwocky est une ballade, une parodie de poème épique et, malgré le caractère déroutant de certains termes, Alice en saisit globalement le sens ( $«[\ldots]$ somebody killed something: that's clear, at any rate»). Remarquons tout de même que les explications fournies par Humpty-Dumpty au chapitre 6 ne portent que sur le premier quatrain et que les quatrains 2 à 6 , qui constituent la narration épique, sont assez compréhensibles. Il serait intéressant de définir ce qui sépare le quatrain 1-7 du reste du poème. Mais nous allons d'abord examiner les éléments qui les unissent, au plan de la connotation, et voir comment les ont traités les traducteurs.

\section{CONNOTATIONS}

Les connotations sémiotiques sont ici des éléments de sens qui, au lieu de s'attacher au déroulement linéaire de l'énoncé, jouent à l'échelle du texte sur divers supports linguistiques. Ces éléments de sens sont multiples, ils se chevauchent et s'entremêlent. Nous avons dû les classer', pour dégager divers signifiés de connotation: l'enfance, l'archaïsme, la poésie épique, le nonsense, le langage. Ces signifiés, qui se recoupent souvent, apparaissent sur les signifiants variés. En même temps que ces signifiés et signifiants, nous avons relevé les questions qu'ils suscitent, tant sur le plan de l'analyse du texte que sur celui de la traduction.

\section{L'ENFANCE}

Ce signifié se manifeste à plusieurs niveaux. Sur le plan textuel, tout d'abord, nous savons que le livre dont est tiré Jabberwocky est destiné à un public d'enfants, public privilégié de Lewis Carroll. Par ailleurs, la parodie introduit un élément ludique, ce qui 
nous renvoie à l'enfance. Le thème de la quête et de la bataille avec le monstre, épreuve initiatique, est un classique des contes et légendes de la littérature enfantine ; le traitement de ce thème est simple, linéaire, sans rebondissements, sans recherche dans le déroulement de l'action.

Les traducteurs suivent fidèlement la chronologie de cette quête. Notons que le poème original est au passé, temps qu'a respecté Parisot, sauf dans le quatrain 5, celui de la bataille, où le présent de narration donne du relief à l'action. Par contre, Warrin a mis tout le poème au présent, ce qui lui confère un caractère intemporel.

Autres éléments à inscrire au registre de l'enfance : une expression comme went snicker-snack (l'emploi de to go + onomatopée est typique du langage enfantin); les répétitions de syllabes, comme dans Jubjub, Tumtum (nombre de mots enfantins se fondent sur de tels jeux phonétiques, qui seront étudiés plus loin) ; enfïn, le nonsense, les mots inventés : nous y reviendrons.

\section{L'ARCHAÏSME}

C'est de diverses manières que ce signifié apparaît. Tout d'abord, Jabberwocky constitue une parodie de ballade médiévale, dont la forme en quatrains, avec des passages lyriques (le quatrain 1-7) est respectée dans les traductions; Warrin reste près du tétramètre, en utilisant l'octosyllabe, alors que Parisot mélange octosyllabes, décasyllabes et alexandrins, ce qui l'éloigne de la forme originale mais enrichit le rythme du poème. Le thème épique de la quête initiatique entre dans ce connoté archaïsant. Par ailleurs, le quatrain 1-7; dans sa version primitive, était présenté comme A Stanza of Anglo-Saxon Poetry dans le journal manuscrit Misch-Masch, que Charles L. Dodgson rédigeait pour amuser ses frères et sœurs. Il était écrit dans une graphie archaiisante: les I étaient remplacés par des Y, les THE par des YE. Repris par la suite pour orner et circonscrire Jabberwocky, le quatrain garde un certain caractère archaïsant malgré sa graphie moderne. Ce même connoté apparaît, sur divers supports, tout au long du poème.

\section{Morphosyntaxe}

- 'Twas (vers 1) : contraction archaïque de It was; signifiant intraduisible.

- brillig (vers 1) : le suffixe anglo-saxon -ig, servant à la formation d'adjectifs, a donné en anglais moderne $-\boldsymbol{y}$; signifiant intraduisible.

- Did gyre and gimble (vers 2); deux éléments : l'emploi de did, qui est perdu à la traduction; l'allitération du $\mathrm{g}$ dans deux verbes séparés par and (il s'agit là d'un procédé poétique ancien, qui sera étudié au chapitre de la phonétique).

- Des inversions: vers 3, All mimsy were the borogoves; vers 10 , Long time the manxome foe he sought; vers 11 , So rested he; vers 13 , In uffish thought he stood. Les traducteurs reprennent certaines de ces inversions: vers 3, Enmîmés sont les gougebosqueux (FW); Tout flivoreux vaguaient les borogoves (HP); vers 10 , Longtemps, longtemps cherchait (HP); ce dernier en ajoute deux: vers 5 , Au Bredoulochs prends bien garde ; vers 12 , Pour réfléchir un instant s'arrêtait.

- Hast thou (vers 21): deuxième personne archaïque ; signifiant intraduisible.

- O frabjous day (vers 23) : exclamation reprise de près par les traducteurs : Ô jour frabbejais (FW) ; Ô jour frableux (HP).

\section{Lexique}

Le lexique archaïsant est pauvre; on peut noter Long time (vers 10); slain, bien qu'appartenant au registre littéraire, peut contribuer au connoté archaissant. Les traducteurs introduisent ce signifié dans la traduction de Beware: Garde-toi (FW); Prends bien garde... gare (HP), et ce dernier ajoute au vers 9 la forme verbale «ceint», 
qui y participe. Il faut toutefois souligner l'importance des termes inventés qui, par leur caractère déroutant, évoquent un dérapage dans le temps.

Les traductions ne peuvent rendre qu'imparfaitement le connoté archaisant ainsi repéré ; celui-ci est toutefois renforcé par le traitement de la poésie épique.

\section{LA POESIE ÉPIQUE}

Thème

La poésie épique est évidemment présente par le thème de la quête initiatique, celle du garçon qui, terrassant le monstre, gagne la reconnaissance du père. Chose intéressante à noter, Carroll ne nomme pas le héros de l'histoire; seul le pronom he et l'adjectif possessif his le mettent en scène, et ils s'appliquent d'ailleurs aussi au père (ambiguïté du vers $24:$ He chortled in his joy). C'est uniquement dans la relation fils-père, manifestée par les apostrophes du père (car le fils, lui, ne parle pas !) : my son, my beamish boy, que se fonde l'existence des deux hommes dans leur complémentarité. Les traducteurs, ici, prennent des positions différentes: Warrin en reste au pronom il (suivant ainsi de très près le texte source), alors que Parisot nomme les protagonistes : «le jeune homme», «le vieux» (on pourrait d'ailleurs lui reprocher la connotation sémantique péjorative introduite par ce dernier terme). Son intervention rapproche le lecteur du plan de la réalité de l'action et, ce faisant, renforce le connoté épique. Mais elle fait également disparaître l'ambiguîté qui, donnant les deux protagonistes comme un seul personnage à double face, renvoyait donc au thème du miroir.

Par ailleurs, nous pouvons aussi penser que Carroll n'a accordé qu'un intérêt superficiel à cette narration, n'y voyant qu'une parodie. Certaines faiblesses de forme peuvent étayer cette opinion : le maigre pouvoir évocateur du quatrain 3, par exemple, ou la répétition inélégante qui apparaît aux vers 12 et 13 . Par opposition, la traduction de Parisot est plus précise et plus évocatrice que l'original en ce qui concerne le connoté épique, et cet aspect se manifeste nettement dans le lexique.

\section{Lexique}

Sur le plan lexical, quelques termes dessinent les étapes de l'action : les monstres, la quête, la bataille, le triomphe.

- Les monstres et le danger qu'ils représentent. Le Jabberwock est le seul qui soit un peu décrit : jaws that bite, claws that catch, eyes of flame; en outre, il est illustré, et sir John Tenniel a dû suivre les indications de l'auteur. Le Jabberwock tient à la fois du griffon et du dinosaure, et il est énorme à côté du frêle jeune homme qui ploie sous le fardeau d'une épée plus grande que lui. Cette illustration fixe des caractéristiques qui n'étaient que sommairement évoquées dans le poème ; toutefois, si l'édition n'est pas illustrée, le traducteur est moins prisonnier des contraintes de l'image. Par ailleurs, le monstre émet des sons: whiffing, burbled. Les traducteurs reprennent l'un et l'autre la griffe et la gueule, parlent d'œil flambant (FW), flamboyant (HP) et inventent des termes pour rendre les sons : siblant, burbule (FW) ; ruginiflant, barigoulant (HP). Les deux autres monstres, Jubjub, Bandersnatch, sont simplement nommés, ce dernier étant qualifié de frumious (traduit par «frumieux» dans les deux cas). Le danger que représentent les monstres est évoqué par les termes beware, shun (garde-toi, évite - FW ; prends bien garde, laisse en paix - HP). Le mot foe, qui représente le Jabberwock, devient le fauve (FW), le monstre (HP); notons que les deux traducteurs ajoutent un nom pour parler du Jabberwock au vers 19 : la bête.

- La quête. C'est le $3^{e}$ quatrain: le départ et la vaine recherche du monstre : sword (épée, glaive); sought (recherche, cherchait); stood in thought (il y reste, 
réfléchissant; pour réfléchir un instant s'arrêtait). Ce quatrain est d'une facture assez faible: le connoté d'une épée précieuse et magique, introduit par vorpal sword, tourne court, la recherche s'essouffle vite avec le maigre pouvoir évocateur des vers de ce quatrain, faiblesse que souligne la répétition des termes in thought / stood aux vers 12 et 13. Les traducteurs ont-ils fait mieux ? Warrin ose un enjambement (va-T-à la recherche, rappel de la chanson enfantine Marlbrough s'en va-t-en guerre), qui nous gêne par son caractère forcé. Parisot choisit l'alexandrin pour les vers 9 et 10 , et répète l'adverbe «longtemps», ce qui évoque la durée de la quête ; remarquons aussi le choix du verbe «ceindre», qui enrichit le connoté épique ; l'adjectif «vorpaline», dont la terminaison, qui accentue l'idée de matière précieuse (opaline) et s'oppose à la brutalité du vor (vorace, vortex?), vient encore renforcer ce connoté. Les deux traducteurs évitent la fâcheuse répétition des vers 12 et 13 .

- La bataille: quelques termes, mais surtout un rythme, des répétitions, des onomatopées; le $5^{\mathrm{e}}$ quatrain est très vigoureux, très sonore (voir l'analyse phonétique). Les deux traducteurs en ont respecté le rythme et obtiennent tous deux un effet d'évocation intéressant.

- Le triomphe. La tête du monstre, symbole de la victoire, est coupée; c'est le galop triomphal du retour (galumphing back). Notons que Parisot ajoute le verbe «brandissant» (vers 19) et, là encore, enrichit le connoté épique. Puis vient l'accueil du père, la reconnaissance de l'exploit (vers 21): Warrin garde 1'interrogation, Parisot opte pour l'exclamation - cela se justifie, puisqu'il s'agit d'une fausse question qui est le constat public d'un haut fait; on note à la fois la démonstration d'affection (Come to my arms: Viens à mon cour - FW; Dans mes bras - HP) et les cris de joie, manifestation bruyante et publique ; les traducteurs, là encore, restent près de l'original, même lorsqu'ils ont affaire à des termes inventés par Carroll.

\section{LE NONSENSE}

C'est le terme anglais que nous reprenons ici, avec ses deux significations: d'une part, le non-sens, refus ou absence de sens; d'autre part, un type de création littéraire ${ }^{3}$. Le nonsense, dans Jabberwocky, joue évidemment à l'échelle du poème, qui s'ouvre et se ferme sur un quatrain incompréhensible, pour lequel Carroll a donné deux explications : la première dans Misch-Masch, la deuxième au chapitre 6 de Through the Looking-Glass, par la bouche de Humpty-Dumpty. Par ailleurs, la parodie de ballade médiévale, le détournement de texte, manifestent une volonté qui participe de ce nonsense. Toutefois, rien, dans la syntaxe du poème ni dans le déroulement de l'action, ne nous semble déroutant. C'est surtout sur le plan lexical que le nonsense se manifeste, encore que de façon inégale au fil du poème, la fréquence des mots nonsensiques ${ }^{4}$ étant beaucoup plus élevée dans le quatrain 1-7 que dans les quatrains 2 à 6 . Ce vocabulaire nonsensique, c'est, d'une part, des mots inventés par Carroll, d'autre part, des mots qu'il a détournés d'une façon ou d'une autre; nous examinerons tout d'abord la structure du poème vidée des termes inventés (les mots détournés sont en gras).

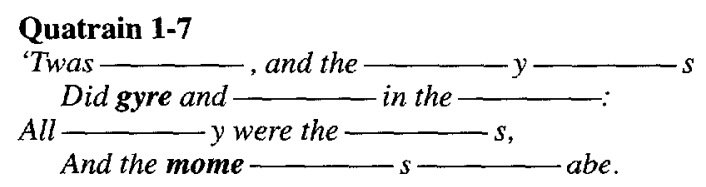

Nous n'avons là qu'un squelette, quelques miettes de sens : des sujets au pluriel, accompagnés d'adjectifs; des verbes au passé; une structure syntaxique parfaitement 
conforme à la norme de la langue; mais les mots pleins ne nous mettent guère sur la piste d'un référent.

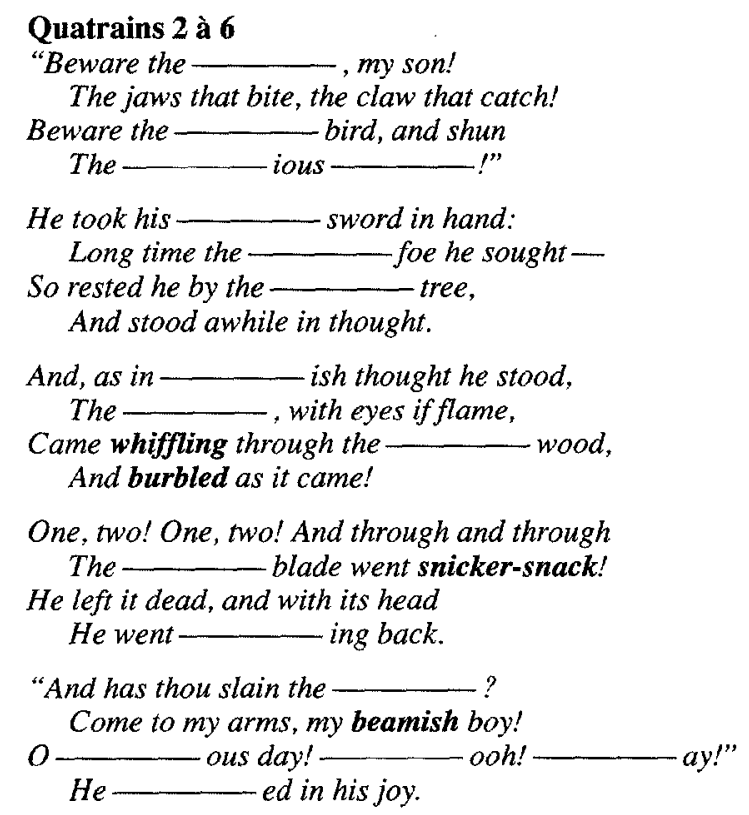

Quelques éléments du lexique nous manquent, mais l'histoire reste très compréhensible, et le mécanisme référentiel global s'établit, comme le dit Alice : nous repérons facilement un thème connu de la poésie épique.

Revenons à ce vocabulaire nonsensique et à la façon dont les traducteurs l'ont traité. Nous le faisons donc entrer dans deux catégories: les mots détournés et les mots inventés, dont nous donnerons quelques exemples.

\section{- Mots détournés}

- gyre, vers 2 : ici, c'est une racine savante qui prend le statut de verbe, avec deux explications différentes $(1$, to scratch like a dog; 2 , to go round and round like a gyroscope) et deux prononciations différentes, [g] puis [dz]. Les traducteurs, suivant sans doute l'explication de Humpty-Dumpty, ont créé le verbe «gyrer»;

- mome, vers 4: nom attesté dans les dictionnaires, auquel Carroll donne la fonction d'épithète et deux explications : «grave»; "from home, meaning that they'd lost their way». Warrin invente «momerade», combinant phoniquement les deux mots mome et raths, et emploie le singulier. Parisot, avec «fourgus», crée un mot-valise;

- Tumtum, vers 11: «a strumming sound», d'après le dictionnaire; les traducteurs se sont attachés seulement au connoté nonsensique et ont exploité le mot à des fins prosodiques (FW, Tété, HP, Tépé);

- burbled, vers 16 : terme attesté, mais pour lequel Carroll invente, dans une lettre à une petite amie, un dénoté de mot-valise; les traducteurs le suivent en inventant eux aussi : «burbule» (FW), «barigoulant» (HP).

Notons que Warrin et Parisot vont plus loin que Carroll : ils traitent comme nonsensique le verbe whiffle: «siblant» (FW), «ruginiflant» (HP) et l'adjectif beamish: «rayonnais» (FW), «rayonnois» (HP). Ce faisant, ils enrichissent le connoté de nonsense. 
- Mots inventés

- brillig, dont nous avons déjà parlé (définition de Carroll: «the time of broiling dinner, i.e. the close of the afternoon»). Warrin, qui ne tient pas compte du dénoté proposé par Carroll, garde l'élément bril- (il brilgue) qui, sémiquement et phoniquement, évoque l'idée de brillant; par contre, Parisot respecte le dénoté (il était reveneure) au détriment de l'aspect phonique. Notons que, dans une version antérieure, il avait créé le mot grilheure qui, gardant le dénoté, maintenait le jeu phonique avec la paronymie bril/gril;

- wabe: c'est «the grass plot round a sundial», comme le devine Alice dans sa conversation avec Humpty-Dumpty («it goes a long way before it, and a long way behind it, $[\ldots]$ and a long way beyond it on each side»), dénoté qu'a repris Parisot en le modifiant légèrement pour créer l'allouinde («l'allée qui mène au cadran solaire [...] elle s'allonge loin devant le cadran solaire, loin derrière lui [...]»). Warrin reste près de l'aspect phonique avec le guave.

- Des noms d'êtres vivants : nous avons, bien sûr, le Jabberwock, et il serait difficile de ne pas y relever jabber ${ }^{5}$; Warrin garde une partie du dénoté avec Jaseroque; il ignore d'ailleurs le suffixe $\mathbf{- y}$ du titre. Parisot, dans ses versions antérieures, garde «Jabberwocky» et «le Jabberwock»; puis il francise le titre: «Jabberwoqueux»; enfin, franchissant le pas, et se rapprochant du thème du langage, il invente le Bredoulochs et compose le titre Bredoulocheux (nous y reviendrons). Quant aux toves, qui sont «something like badgers [...] something like lizards [...] something like corkscrews», ils sont ainsi représentés par Tenniel; ils restent des toves chez Parisot et des tôves chez Warrin. Alors que Parisot garde les borogoves («something like a live mop»), Warrin invente les gougebosqueux (sans doute en cherchant un dénoté: gouge pour bore, et bosqu- pour grove, qui ressemble à gove? En ce qui concerne raths (deux explications de Carroll: «a kind of land turtle»; «a sort of green pig»), Parisot crée un mot-valise à partir de ce deuxième dénoté (verchon $=$ vert + cochon), alors que Warrin colle au connotant phonétique pour lier en un nom l'adjectif et le nom de départ (mômerade).

Nous examinerons aussi du point de vue phonétique les noms $J_{u b j u b}$ et Tumtum. Quant au Bandersnatch, Warrin colle une fois de plus à l'original en parlant de «Band-àprend». Parisot crée le «Pinçmacaque», qui reprend snatch de façon plaisante et y ajoute un connoté exotique et coloré avec le dénoté de «macaque».

- Des adjectifs : slithy, mimsy, frumious, uffish sont des mots-valises pour lesquels, dans Misch-Masch, au chapitre 6 de Through the Looking-Glass, dans la preface de The Hunting of the Snark ou dans ses lettres, Carroll donne des explications qui établissent un dénoté mais, tout en créant un certain cadre, laissent tout de même au traducteur le loisir d'inventer. Pour les adjectifs vorpal, manxome, tulgey, frabjous, qui ne sont pas expliqués, cette liberté pourrait être plus grande encore, mais nous observons que les traducteurs se sont attachés à en respecter la forme, c'est-à-dire le connotant phonétique.

- Des verbes : mis à part gimble («to make holes like a gimlet»), traduit par les verbes «vriller» (FW) et «vribler» (HP), et outgrabe («something between bellowing and whistling, with a kind of sneeze in the middle»), traduit par «horsgrave» (FW) et «bourniflaient» (HP, qui tient compte ici encore du dénoté), ce sont des mots-valises: galumphing (gallop + triumph), traduit par «gallomphant» ou «galomphant»; chortled (chuckle + snort); Warrin colle à la forme (cortule); Parisot crée ce qui semble être un mot-valise (glouffait $=$ glousser + pouffer $?$ ).

Nous relevons que, en général, les traducteurs sont allés plus loin que l'auteur dans l'invention et le nonsense. Parisot pratique par exemple une inversion au vers 6 en 
intervertissant griffe et gueule, et ajoute un verbe de son cru, «lourmait», au vers 13, pour introduire les «suffèches pensées». Ces procédés enrichissent le connoté de nonsense.

Ce connoté est d'ailleurs présent de façon inégale au fil du poème, et la fréquence des mots nonsensiques est beaucoup plus élevée dans le quatrain 1-7 que dans les quatrains 2 à 6 . C'est donc le premier quatrain qui porte la plus grande partie du nonsense; c'est aussi le quatrain le mieux connu du poème. Le connoté mystérieux qu'il dégage a stimulé bien des imaginations. Les explications fournies, souvent à posteriori, par Carroll, et le référent qu'il crée ainsi, n'ont que peu de poids; un «borogove» est peut-être pour l'auteur «a live mop», c'est aussi beaucoup plus dans l'imagination du lecteur - sa lecture est une autre «traduction» - qui laisse Carroll à son interprétation pour créer son propre nonsense. Nous verrons plus loin que d'autres écrivains et traducteurs, fascinés par ce quatrain, en ont fait des lectures diverses.

\section{LE LANGAGE}

Nous ne pouvons échapper, dans la recherche des connotations, à la question du langage, thème central de l'œuvre de Carroll, d'autant que le monstre du poème incarne par son nom la difficulté de la communication (jabber : difficulté de l'émission et de la compréhension du message). Nous nous contenterons de relever quelques éléments qui intéressent le traducteur. D'une part, la traduction du nom du monstre doit évoquer ce problème de communication. Ensuite, nous avons remarqué que le jeune homme ne parle pas. D'autre part, le poème comporte bon nombre de redoublements, ce que nous avons rapproché du connoté de l'enfance, mais qui nous renvoie à un élément autobiographique : $\mathrm{Ch}$. Dodgson était bègue.

C'est peut-être de cet aspect qu'a tenu compte Parisot dans sa dernière version lorsqu'il choisit de baptiser le Jabberwock «Bredoulochs»; nous pouvons y voir d'une part «bredouiller» (la difficulté d'élocution), d'autre part [loc], qui renvoie au latin loqui, parler; donc une double référence au langage, et une répétition d'ordre sémantique. En même temps, au vers 21 , il introduit une répétition d'ordre phonétique qui est presque un bégaiement: «tu as tué» $[/$ ty/a/ty/e/]. Nous pourrions proposer l'interprétation suivante : le fils, tuant le monstre, s'est rendu maître du langage; c'est maintenant le père qui se met à bégayer?

Cet exemple nous permet de conclure au rôle actif du traducteur qui prend nécessairement position, renforce un connoté plutôt qu'un autre. Mais de quelle marge de mancuvre dispose-t-il réellement? C'est peut-être l'analyse phonétique qui, définissant la texture phonique du poème, va établir les repères nécessaires parmi lesquels la traduction pourra se structurer.

\section{ANALYSE PHONÉTIQUE}

Nous avons analysé le poème du point de vue phonétique dans le but de déterminer, en premier lieu, si certains sons dominaient, s'ils étaient expressifs (c'est-à-dire s'ils contribuaient à créer une atmosphère, à communiquer des sentiments ou des émotions) et s'ils constituaient, par le fait même, un support de connotation.

Certains procédés stylistiques relevant de la phonétique ont aussi un effet expressif qu'il est important de chercher à reproduire au moment de la traduction, pour conserver la tension interne qui est un des critères de la poésie (voir Osers 1978: 14). Nous avons donc, en second lieu, identifié les procédés stylistiques auxquels Lewis Carroll a eu recours et nous avons comparé les deux versions françaises du poème pour déterminer dans quelle mesure les traducteurs s'étaient conformés à l'original sur le plan phonétique. 


\section{L'EXPRESSIVITÉ DES PHONÈMES}

Nous avons d'abord compté les phonèmes dans toutes les positions (initiale, médiane et finale) et nous avons noté que les occlusives étaient plus nombreuses que les autres types de consonnes (spirantes, liquides). L'analyse séparée de chaque quatrain a démontré que $[\mathrm{b}, \mathrm{g}, \mathrm{t}]$ avaient le plus grand nombre d'occurrences et que leur emploi répété créait une certaine atmosphère que le traducteur pourrait essayer de rendre à l'aide de phonèmes de même nature dans la langue cible. Ainsi, dans le premier quatrain, l'occlusive sonore [g] est employée à cinq reprises; son articulation dure, combinée avec les voyelles graves $[0, a r, a]$, évoque une atmosphère sombre. Le deuxième quatrain donne une impression tout à fait différente : ici, c'est plutôt d'un geste articulatoire qu'il s'agit. En effet, la bi-labiale [b] est renforcée par les [m] et les [w] qui ont le même point d'articulation; le mouvement des lèvres qui s'avancent pour former les voyelles arrondies [y] et $[u]$ et qui se rejoignent pour former les consonnes bi-labiales crée une moue d'insistance qui souligne les paroles du père mettant son fils en garde contre les dangers qui l'attendent. Les mêmes consonnes sont reprises dans le sixième quatrain; c'est encore le père qui parle, qui s'exclame de joie cette fois devant l'exploit de son fils. On retrouve sur le plan phonétique la symétrie formelle relevée dans la structure du poème.

L'occlusive sourde [t] apparaît neuf fois dans le troisième quatrain. Selon M. Grammont (1960 : 387), les occlusives sourdes suggèrent un bruit sec, momentané, et leur répétition donne l'impression que le bruit est répété. De prime abord, la relation entre le phonème répété et le sens du poème (le jeune homme se repose après avoir longtemps cherché le Jabberwock) n'est pas évidente. Toutefois, on peut imaginer que le cour du héros bat à coups redoublês parce qu'il s'est tant dépensé ou parce qu'il appréhende la rencontre prochaine avec le monstre. Mais ce phẻnomène peut aussi nous renvoyer à la question du bégaiement.

En comparant les deux traductions, nous avons noté que, dans le premier quatrain, Warrin a cherché à conserver les [g], les [m] et les [b], alors que Parisot a introduit quantité de $[\mathrm{f}]$ et de $[\mathrm{v}]$; ces consonnes sont des continues et elles donnent une impression de calme et de sérénité qui ne correspond pas à celle du poème anglais. En revanche, au vers 22 , il a gardé trois des phonèmes importants $[\mathrm{m}, \mathrm{b}, \mathrm{m}]$ tandis que Warrin n'en a conservé qu'un. Nous suggérons de remplacer rayonnois (HP) et rayonnais (FW) par miroitois ou marvoullois, ou tout autre adjectif commençant par [m]. Quant aux [t] du troisième quatrain, Parisot en a conservé cinq et Warrin quatre. Nous proposons le verbe méditer pour remplacer réfléchir et, dans la version de Warrin, d'utiliser monstre au lieu de fauve: ce terme était bien choisi pour rendre foe phonétiquement, mais il désigne les grands animaux féroces, les félins surtout, ce qui ne correspond pas à la bête illustrée par John Tenniel.

\section{LES PROCÉDÉS STYLISTIQUES}

Lewis Carroll a utilisé plusieurs procédés stylistiques à caractère phonétique qui donnent du rythme au poème et le colorent d'impressions variées. Ce sont:

Lalliterative meter: «A prominent feature of Old Germanic prosody is initial rhyme, used not for mere ornamentation but structurally, to link and emphasize important words within prescribed metrical units. [...] The fondness for alliterative formulas is still noticeable in a language like English, which uses them easily and habitually in and out of poetry: "might and main", "time and tide", "to hide and to hold".» (Preminger 1965). Un exemple d'alliterative meter se trouve dans le premier quatrain: «Did gyre and gimble in the wabe.» Les traducteurs n'ont pas conservé l'allitération, aussi une correction serait-elle justifiée ici. Devant la difficulté à trouver deux termes avec un [g] initial et qui serait sémantiquement acceptables, nous avons opté pour le maintien des 
verbes vriller (FW) et vrible (HP) et nous proposons virer comme premier terme. En outre, dans la version de Warrin, nous mettrions tous les verbes à l'imparfait, pour respecter la structure métrique et, pour la même raison, nous remplacerions enmîmés par mismeux, mot-valise fait de misérable et malheureux. Le quatrain de Warrin, corrigé, se lirait donc comme suit :

Il brilguait: les tôves lubricilleux.

Viraient et vrillaient dans le guave,

Mismeux étaient les gougebosqueux,

Et le mômerade horsgrave.

Le redoublement : «Le redoublement consiste dans la répétition sans changement d'un mot ou d'une syllabe.» (Grammont 1960: 380) Il y a plusieurs exemples de redoublement dans le poème : vers 5 et 7 :Beware - Beware; vers 17, One, two!, One, two!, And through and through! (redoublement de mot); Jubjub, vers 7, et Tumtum, vers 11 (redoublement de syllabes).

Le redoublement de mots a un effet d'insistance qu'il faut conserver dans la traduction. Il a été très bien rendu par Warrin: Garde-toi - Garde-toi, mais non par Parisot: Prends bien garde - Gare, quoique ce dernier ait su conserver l'allitération. En revanche, Parisot a bien traduit le second exemple (pour conserver le parallélisme, il faudrait toutefois éliminer fulgurant); Warrin l'a traduit par un redoublement et une rime intérieure (voir plus loin), ce qui a certainement autant d'effet.

Le redoublement de syllabes a une valeur insistante; il renvoie encore au bégaiement, mais surtout il rappelle le langage enfantin, qui est très marqué par l'emploi de disyllabes composées par la répétition d'une même syllabe et servant à nommer des personnes, des animaux ou des objets familiers (Grammont 1960: 380). Comme Lewis Carroll écrivait pour des enfants, on peut croire que son intention était d'imiter leur langage lorsqu'il a créé les noms de l'oiseau Jubjub et de l'arbre Tumtum. Il y a donc lieu de conserver la répétition, et c'est ce qu'a fait Parisot. Warrin écrit l'oiseau Jube : si la structure en tétramètres ne lui permettait pas le redoublement, il aurait pu composer le nom avec une consonne géminée, qui a aussi un effet d'insistance et qui est une autre caractéristique du langage enfantin (Grammont 1960:382). En outre, la voyelle [y] est antérieure, haute et tendue; elle ne correspond pas à celle de l'anglais, qui est postérieure, moyenne (mid) et relâchée $[\Lambda]$. Il aurait donc pu écrire Jobbe. Parisot a établi une assez bonne correspondance avec Jeubjeub. Dans les deux cas, l'ajout d'un trait d'union rendrait le mot plus conforme à la morphologie du français, et une majuscule à l'initiale indiquerait qu'il s'agit du nom de l'oiseau.

En ce qui a trait au mot Tumtum, les deux traducteurs ont répété la voyelle, mais encore une fois, le timbre ne correspond pas à celui de la voyelle en anglais. Le choix de la voyelle [e] a probablement été dicté par le souci de conserver la rime intérieure :

(E) So rested he by the Tumtum tree

(F) Puis arrivé à l'arbre Té-Té (Tépé)

Nous faisons donc la proposition suivante, qui respecterait les deux procédés et, en outre, conserverait la voyelle longue de l'anglais :

Après un somme sous l'Arbre-Tomme,

Il y reste, méditant.

L'assonance est l'identité ou l'analogie de voyelles proches en position initiale ou dans les syllabes accentuées (Shapiro et Beum 1965: 95). On en trouve quelques exemples aux vers 6 (jaws - claws), 9 (vorpal sword), 10 (long - manxome: deux 
voyelles nasales), et 19 (left-dead). Dans le premier exemple, les deux traducteurs ont reconnu l'effet d'insistance de la répétition du son vocalique et l'ont rendu par la répétition de la consonne $[\mathrm{g}]$ : griffe - gueule, en ayant recours à la compensation.

Warrin n'a pas tenu compte des assonances dans les deuxième et troisième exemples, mais il a réussi des assonances très riches au vers 19 , où les consonnes qui suivent la voyelle faisant assonance sont identiques. Parisot n'a pas créé d'assonance au vers 9 , mais il a compensé cette lacune sur le plan lexical, comme nous l'avons indiqué dans la première partie. Par ailleurs, au vers 10 , il a employé six voyelles nasales, et au vers 19 , il a aussi réussi une assonance avec la voyelle [ ${ }^{\natural}$ ].

L'allitération diffère de l'alliterative meter dont nous avons discuté plus haut en ce qu'elle est employée pour créer un effet esthétique. Elle est la répétition d'un son ou d'une syllabe dans deux mots (ou plus de deux mots) d'un même vers ou d'un groupe de vers. Le type d'allitération le plus fréquent est la répétition des sons initiaux, plus particulièrement des consonnes ou groupes de consonnes. L'allitération inclut aussi la répétition de consonnes, de voyelles, ou de groupes consonne-voyelle en position médiane ou finale6.

Dans Jabberwocky, nous avons noté des allitérations aux vers 11, Tumtum tree; 22, beamish boy; 23, Callooh! Callay!; 24, chortled_-joy. Dans le premier exemple, il y a collision de deux procédés dans le vers, l'allitération et la rime intérieure (dont nous parlerons au paragraphe suivant), et les deux traducteurs ont opté pour la conservation de cette dernière. Pour beamish boy, nous avons suggéré marvouillois pour remplacer les traductions littérales: rayonnais (FW) et rayonnois (HP). Quant à l'allitération de Callooh! Callay!, elle a été respectée dans les deux versions. Dans le dernier exemple, les consonnes initiales de chortled et de joy ont le même point d'articulation, et nous proposons de modifier légèrement la traduction de Warrin de manière à respecter cette similitude; nous écrirons donc chortule au lieu de cortule. Parisot a créé un mot-valise (glouffait) qui tient compte de l'aspect sémantique plutôt que de l'aspect phonétique.

La rime intérieure. Selon Shapiro et Beum (1965: 89), la rime intérieure, jusqu'à tout récemment, était toujours construite sur le même modèle : un mot près du milieu, ou au milieu du vers, rimait avec le dernier mot du vers. Quatre vers du poème illustrent ce procédé :

a) So rested he/by the Tumtum tree: qui a déjà été commenté plus haut dans le paragraphe sur le redoublement;

b) One, two! One, two!/And through and through!: deux procédés ont été utilisés dans le même vers: le redoublement et la rime intérieure. L'un des traducteurs (Parisot) a choisi de conserver le redoublement, l'autre a traduit avec la rime intérieure;

c) He left it dead / and with its head: la traduction est heureuse dans les deux versions;

d) O frabjous day!/Callooh! Callay!: Au lieu de créer une rime intérieure, Parisot a fait rimer ce vers avec le premier vers du quatrain, ce qui lui donne de l'unité rythmique et plus d'intensité. Quant à Warrin, il a respecté la rime intérieure et il a également fait rimer les vers 22 et 23 du même coup. Mais cette solution n'est valable que pour les lecteurs pour qui la graphie ais $=[\mathrm{e}]$. C'est pourquoi nous proposons de modifier le vers de deux façons: nous transformons frabbejais en frabieux (par analogie avec frumieux, vers 8) qui est une traduction phonétique, mais c'est un procédé auquel Warrin a eu recours plusieurs fois, et nous remplaçons Calleau! Callai ! par Taïoux! Taïeux! plus français parce qu'il rappelle le cri de chasse Taïaut! La version de Warrin se lirait donc comme suit: 
As-tu tué le Jaseroque?

Viens à mon cour, fils marvouillois !

Ô jour frabieux ! Tä̈oux ! Taïeux!

Il chortule dans sa joie.

L'onomatopée et l'harmonie imitative. Il y a lieu de faire une distinction entre l'onomatopée et l'harmonie imitative. La première est l'imitation fidèle, «la reproduction plus ou moins exacte de bruits, de cris existant dans la nature» (Grammont 1947: 203); la seconde est le procédé par lequel les poètes essaient de «peindre certains bruits, d'en donner à l'oreille l'impression par les sons des mots qu'ils emploient» (Grammont 1947 : 196).

Les mots de formation onomatopéique sont habituellement constitués par le redoublement d'une syllabe (glouglou, ronron). Dans d'autres cas, ils obéissent à une règle de formation comportant une apophonie (ou alternance vocalique) spéciale qui veut que «leurs voyelles accentuées soient d'une manière générale i, a, ou, allant de la plus claire à la plus sombre, sans que cet ordre puisse être interverti. Quelquefois l'a est remplacé par un o ouvert de valeur à peu près équivalente. [...] Ex. : pif-paf, pif-pafpouf, flic-flac, cahin-caha ; en anglais : ping-pong, jingle-jangle» (Grammont 1960: 380-381). Il faut noter, en outre, que les consonnes sont répétées même si la voyelle change.

L'onomatopée que Lewis Carroll a créée pour imiter le bruit de l'épée qui frappe le monstre à grands coups est conforme à cette apophonie (qui est commune à plusieurs langues), et nous estimons qu'il est important d'utiliser les mêmes sons en traduisant, ou tout au moins la même apophonie. Nous proposons donc de traduire snicker-snack par vlin-vlan, qui respecte les règles que nous venons de mentionner, qui maintient la rime avec galomphant, et qui fait allitération avec glaive vorpal(in).

Pour ce qui est de l'harmonie imitative, Carroll a peint les bruits que fait le monstre surgissant de la forêt: whiffling, avec [wh] et le [f] géminé, suggère le souffle violent de l'animal, et burbled, avec ses [b] répétés, les deux liquides [l] et [r] et l'occlusive [d] à la fin, suggère de la turbulence, un bruit de liquide, comme si l'animal écumait et crachait à chaque expiration. Les solutions de Parisot sont bien trouvées: ruginiflant, avec la spirante [f] et ses quatre syllabes, donne l'impression du souffle qui se prolonge ; le [r], qui souvent exprime un grondement, suggère que le souffle est exhalé avec bruit. Barigoulant a conservé les consonnes principales [b, $\mathrm{r}, \mathrm{l}]$ et le $[\mathrm{g}]$ peut même faire penser que le bruit vient du fond de la gorge. Warrin est un peu plus faible sur ce point : son burbule est une traduction phonétique qui reprend les phonèmes du texte de départ, mais il manque un peu de force. Nous proposons d'intercaler un [y] entre [r] et [b] (burubule) pour faire ressortir le [r] et pour accentuer l'impression du liquide qui sort en bulles. Siblant n'évoque que de loin un sifflement (sibilant); il pourrait être remplacé par vifflant, qui serait une équivalence phonétique; 1 'articulation de $[\mathrm{v}]$ est assez près de celle de [wh], et [fl] donne l'impression du souffle qui précède l'apparition de la bête.

Quelques remarques générales sur l'ensemble du poème et sur les mérites respectifs de chaque traduction serviront de conclusion à cette analyse phonétique. Tout d'abord, sur le plan des consonnes, la prépondérance d'occlusives, dont l'articulation s'accompagne d'une légère explosion, donne un mouvement saccadé au déroulement du poème. Le rythme est aussi accentué par la structure de chaque quatrain - trois tétramètres et un trimètre - qui est répétée dans tout le poème. L'impression de mouvement s'intensifie au cinquième quatrain, dans lequel l'auteur a accumulé les procédés stylistiques : redoublement, rime intérieure à deux reprises et onomatopée se combinent pour illustrer d'une manière très vivante la bataille qui se déroule. La fréquence des occlusives confère une certaine unité phonétique au poème, ce qui devrait 
inciter le traducteur devant faire un choix de traduction à privilégier un terme comprenant ce type de consonnes. En revanche, les voyelles ne dessinent aucun schème significatif et viennent contredire cette impression d'unité.

En second lieu, Warrin a eu le souci de conserver la structure métrique, ce qui donne plus d'intensité à sa version. En effet, si l'on suit Schneider (1978: 30), «la traduction se doit de respecter le disposition en strophes, la longueur des vers. On n'a pas le droit de traduire un vers court par un vers notablement plus long. [...] Le poème perdra de sa densité». C'est ainsi qu'au cinquième quatrain, même si les deux traducteurs ont tous les deux bien rendu les procédés stylistiques, la version de Warrin a plus de force parce que les vers sont plus courts.

Dans l'ensemble, Warrin a été plus fidèle que Parisot à l'aspect phonétique du texte original, mais c'est au prix d'une certaine faiblesse sur le plan de l'originalité. Il a traduit phonétiquement, et parfois littéralement, les mots inventés par Lewis Carroll, alors que Parisot a fait preuve de créativité, privilégiant l'aspect sémantique à l'aide des indications données par l'auteur.

\section{CONCLUSION}

Quelques éléments se détachent au terme de cette étude, tant au sujet du poème qu'à propos des traductions qui en ont été faites.

Les traductions examinées plus haut présentent des différences très marquées.

Frank $\mathrm{L}$. Warrin a suivi de très près la forme du texte original, sauf en ce qui concerne la question du temps des verbes. Il en a respecté la métrique, il a privilégié les aspects phonétiques, au détriment parfois du contenu sémantique et des connotations, et son poème, dans sa fidélité formelle, perd en expressivité.

Henri Parisot, quant à lui, a pris nettement position en se démarquant souvent de l'original. De façon générale, nous pouvons dire qu'il choisit d'adapter le poème à son destinataire : le lecteur enfant. À cette fin, il nomme les protagonistes et enrichit certains connotés, surtout celui de la poésie épique et du nonsense, en ajoutant des termes qui donnent plus de précision au lexique, en inventant des mots-valises ou en introduisant des effets syntaxiques, ce qui confère à son poème un grand pouvoir d'évocation. Par ailleurs, il suggère la problématique du langage, thème central chez Lewis Carroll.

Jabberwocky a inspiré bien des auteurs, mathématiciens, logiciens, linguistes, écrivains de science-fiction et, bien sûr, traducteurs. Nous retiendrons la version du premier quatrain que nous ont donnée Boris Vian et Antonin Artaud.

Boris Vian, grand traducteur, a traduit la remarquable nouvelle de science-fiction All Mimsy Were the Borogoves (Tout smouales étaient les Borogoves) de Lewis Padgett. Voici sa version du quatrain 1-7:

Lfut bouyeure et les filuants toves

Gyrèrent et gilbèrent dans la loirbe...

Tout smouales étaient les borogoves

Et les dcheux verssins hurliffloumèrent ${ }^{7}$...

Dans cette nouvelle, le quatrain est la clé d'une autre dimension, une formule mathématique parfaite, envoyée du futur par une machine à voyager dans le temps, et que deux enfants parviennent à déchiffrer pour disparaitre dans la quatrième dimension. Vian l'interprète dans l'optique de l'ésotérisme, notamment en jouant avec le temps des verbes : alors que Warrin emploie le présent et Parisot l'imparfait, Vian fait alterner passé simple et imparfait. La présence du passé simple aux vers 1 et 2 crée une tension; l'imparfait du vers 3 représente peut-être une condition à remplir; enfin, le passé simple du vers 4 peut marquer le grand départ des deux («dcheux»?) enfants qui s'évanouissent 
en fumée. À la fin de la nouvelle, le père des enfants, resté seul, s'interroge sur le nonsense, la démence, et se demande s'il ne pourrait pas, en devenant fou, suivre ses enfants. Mais non. «Ce serait la mauvaise espèce de folie.» Cette folie-là, la «mauvaise», nous renvoie pourtant à un autre traducteur de Lewis Carroll, Antonin Artaud.

Artaud a traduit le chapitre 6 de Through the Looking-Glass, dans le cadre de son art-thérapie, et c'est là qu'il donne sa version du premier quatrain de Jabberwocky :

Il était Roparant, et les vliqueux tarands

Allaient en gibroyant et en brimbulkdriquant

Jusque-là où la rourghe est à rouarghe à rangmbde et rangmbde à rouarghambde :

Tous les falomitards étaient les chats-huants

Et les Ghoré Uk'hatis dans le GRABUGEUMENT ${ }^{8}$.

Il correspond à ce sujet avec Henri Parisot (voir exergue). Si «les deux premiers vers répondent encore aux critères de Carroll, $[. .$.$] dès le dernier mot du second vers, dès$ le troisième vers, un glissement se produit [...]. Avec effroi, nous le reconnaissons sans peine : c'est le langage de la schizophrénie» (Deleuze 1969: 102-103). Dans l'ensemble du chapitre, la rencontre Alice/Humpty-Dumpty reste reconnaissable, malgré de fréquents effondrements, des dérapages : par exemple, le titre du poème, Jabberwocky, devient une série de «phrases» de quatre ou huit syllabes, parmi lesquelles il faut choisir (par exemple «MOMAR UNI» Ou «GONTAR ARAK ALAK ELI»). Artaud entretient avec Carroll des relations extrêmement contradictoires : il s'identifie à son ouvre au point d'accuser Carroll de l'avoir plagié : «J'ai eu le sentiment [...] que ce petit poème c'est moi qui l'avais pensé et écrit, en d'autres siècles, et que je retrouvais ma propre œuvre entre les mains de Lewis Carroll.» Mais il lui reproche d'être, selon le mot de Deleuze, «un petit pervers qui s'en tient à l'instauration d'un langage de surface et n'a pas senti le vrai problème d'un langage en profondeur - problème schizophrénique de la souffrance, de la mort et de la vie». C'est la surface qui fait problème chez les schizophrènes; c'est à la surface que le mathématicien-écrivain Dodgson tisse les réseaux logiques qui structurent son rapport avec le monde, et c'est cette surface que structure le langage. Artaud, dans son entreprise personnelle de déstructuration du langage, recrée l'œuvre de l'intérieur, la détruisant pour se l'approprier.

Notons que Vian (traduisant Padgett), et surtout Artaud, en retenant seulement le premier quatrain, éliminent la narration épique, ce qui nous amène à poser de nouveau la question de l'unité du poème. Le caractère artificiel de la juxtaposition du quatrain 1-7 aux quatrains 2 à 6 est historiquement indéniable, mais Carroll a-t-il pu donner une unité à l'ensemble? Sur le plan lexical pas plus que sur le plan syntaxique, nous n'avons pu relever d'unité. Toutefois, celle-ci peut se manifester sur le plan sémiotique, par certains connotés: l'archaïsme, le nonsense; sur le plan phonétique, nous avons noté une constante dans les consonnes: la fréquence des occlusives; par contre, les voyelles ne dessinent aucune structure repérable. Ainsi, selon l'aspect qu'il aura privilégié, le lecteur pourra insister sur la présence ou l'absence d'unité.

Pour finir, nous nous poserons la question de la traduisibilité. Jabberwocky peut sembler intraduisible au premier abord; signalons qu'André Bay et Jacques Papy, qui ont traduit Through the Looking-Glass, n'ont pas touché au poème et ont utilisé des versions de Henri Parisot. Ce dernier a lui-même retravaillé nombre de fois son texte, y apportant des modifications mineures mais qui montrent les choix auxquels il a été confronté. Nous avons nous-mêmes proposé quelques modifications aux traductions pour accentuer certains aspects phonétiques, mais sans y aller de notre propre version du poème.

Pourtant, notre étude vient infirmer l'impression d'intraduisibilité de la première lecture; tout au contraire, malgré son caractère sommaire, elle fait ressortir une foule 
d'éléments dont le décryptage ouvre des perspectives diverses. Le traducteur, dans ce tulgey wood du poème, va prendre position, privilégiant, par contrainte ou par choix, certains aspects par rapport à d'autres.

Jabberwocky est un texte dont la charge poétique, la tension restent faibles. Les aspects les plus évocateurs (nonsense, poésie épique) cèdent vite à l'analyse et viennent facilement sous la plume du traducteur. D'autres, plus subtils, comme le thème du langage, demandent une lecture plus approfondie. La liberté du traducteur est grande: c'est celle du lecteur, qui plaque sa propre grille d'interprétation sur le texte; mais le lecteur est multiple, et les grilles de lecture nombreuses. Le traducteur de Jabberwocky peut, comme Warrin, suivre très fidèlement la forme du poème ou, comme Parisot, choisir ses destinataires (les enfants, les linguistes?) et, dans le réseau de contraintes que lui imposent les langues de départ et d'arrivée, recréer le discours à leur intention.

Pour illustrer ces diverses approches, nous proposerons simplement deux nouvelles traductions pour le nom du Jabberwock:

- Jaccaroc: les deux premières syllabes de jacasser (déf. : parler avec volubilité et d'une voix criarde, Robert, 1984) qui serait l'équivalent de jabber, deux consonnes géminées pour donner un effet d'insistance, et roc par analogie avec «l'oiseau appelé roc» du conte Sindbad le marin, tiré des Contes des mille et une nuits (ce serait la version pour enfants...).

- Jargouloc: sur le plan phonétique, nous suivons au plus près la première et la dernière syllabes; sur le plan sémantique, nous imitons Parisot en évoquant les problèmes de communication et le langage (renvoi à jargon et à loqui); la voyelle [y] permet un renvoi sémantique à la goule (sorte de vampire des contes orientaux), qui est déjà présente dans le barigoulant de Parisot; d'où renforcement du connoté et lien intratextuel (ce serait la version pour linguistes...).

Notes

1. Voir Barthes, Kerbrat-Orecchioni, Ladmiral.

2. C'est ici qu'apparait l'arbitraire de notre emploi de la connotation: nos critères de définition des connotés ont un caractère subjectif. Répétons qu'il ne s'agit ici que d'utiliser un outil.

3. Définition: «being a poem or other literary composition of humorous or whimsical character [...] often marked by the use of words coined for the purpose that sometimes have an evocative character but no precise or generally accepted meaning» (Webster).

4. Voir Gattegno, 1974

5. Définition: «to talk rapidly, indistinctly or unintelligibly; utter gibberish or nonsense» (Webster).

6. Voir Preminger.

7. Voir Padgett.

8. Artaud, «L'Arve et l'Aume, tentative anti-grammaticale contre Lewis Carroll», L'Arbalète, no 12,1947 , in Euvres complètes, tome IX, p. 165.

\section{BIBLIOGRAPHIE}

ARTAUD, A. (1971): CEuvres complètes, tome IX, Paris, Gallimard.

BARTHES, R. (1970): Le Degré zéro de l'écriture. Éléments de sémiologie, Paris, Éditions du Seuil, coll. «Médiations».

BEAUDIN, H. (1966): Boris Vian, Paris, Éditions du Centurion.

CARROLL, L. (1960): The Annotated Alice, édité par M. Gardner, New American Library.

CARROLL, L. (1961): De l'autre côté du miroir, Paris, Gallimard, «Folio Junior».

CARROLL, L. (1963): Alice au pays des merveilles, Marabout géant illustré, Verviers, Belgique, Éditions Gérard \& $\mathrm{Co}^{\circ}$

CARROLL, L. (1971): Through the Looking-Glass - De l'autre côté du miroir, version bilingue, AubierFlammarion.

CLAS, A. (1980): Sons et langage, Initiation à la phonétique, La Librairie de l’Université de Montréal.

DELEUZE, G. (1969): Logique du sens, Paris, Éditions de Minuit.

GARY-PRIEUR, M.-N. (1971): «La notion de connotation(s)» in Littérature, n 4, décembre 1971. 
GATTEGNO, J. (1974): Lewis Carroll, une vie, Paris, Seuil.

GRAMMONT, M. (1947): Le Vers français, $2^{\mathrm{e}}$ édition, Paris, Librairie Delagrave.

GRAMMONT, M. (1960): Traité de phonétique, 6e édition, Paris, Librairie Delagrave.

KERBRAT-ORECCHIONI, C. (1977) : La Connotation, Presses universitaires de Lyon.

LADMIRAL, J.-R. (1979): Traduire: théorèmes pour la traduction, Paris, Payot.

OSERS, E. (1978): «Some Aspects of the Translation of Poetry», in Meta 23-1, pp. 7-19.

PADGETT, L. (1964): «Tout smouales étaient les Borogoves» in Les Vingt Meilleurs Récits de science-fiction, édité par JUIN; H., Marabout.

PÉRALDI, F. (1973): «Pour traduire: traduction-transconnotation», in Sociologie et sociétés, Presses de l'Université de Montréal, vol. 5, no 2, novembre 1973.

PÉRALDI, F. (1978) : «Pour traduire un coup de dés», in Meta 23-1, pp. 109-123.

PREMINGER, A. (éd.) (1965): Encyclopedia of Poetry and Poetics, Princeton, New Jersey, Princeton University Press.

SCHNEIDER, A. (1978): «La traduction poétique», in Meta 23-1, mars 1978, pp. 20-36.

SHAPIRO, K. et R. BEUM (1965) : A Prosody Handbook, New York, Harper \& Row, Publishers.

SLOAT, C., TAYLOR, S. H. et J. E. HOARD (1978): Introduction to Phonology, Englewood Cliffs, N. J., Prentice Hall, Inc.

WEBSTER (1981): Third New International Dictionary of the English Language.

YAGUELLO, M. (1981): Alice au pays du langage, Paris, Éditions du Seuil. 